\title{
Exploring Sub-Array Strategies for MeerKAT-VLBI
}

\author{
Nkululeko S. Qwabe* \\ South African Radio Astronomy Observatory (SARAO) \\ University of Pretoria \\ E-mail: nqwabeeska.ac.za
}

\section{Roger P. Deane}

University of Pretoria

Rhodes University

E-mail: roger.deane@up.ac.za

MeerKAT is a South African radio interferometer that will be the most sensitive in its class until the operation of the Square Kilometre Array mid-frequency array. Like SKA1-mid, MeerKAT's antennas are configured in a dense core (driven primarily by pulsar and HI science), as well as more extended spiral arms to provide higher angular resolution imaging. The inclusion of the MeerKAT array into global VLBI networks would add significant sensitivity to existing VLBI networks, especially at the longest baselines $(>7000 \mathrm{~km})$. Moreover, this will strengthen the role of the Hartebeesthoek Radio Astronomy Observatory (HartRAO) in VLBI arrays through improved long-baseline calibration. MeerKAT-VLBI will extend VLBI coverage in the southern hemisphere which will be expanded further by the upcoming African VLBI Network (AVN). MeerKAT's ability to be split into several sub-arrays and simultaneously generate interferometric and tied-array data products provides the opportunity to further increase its high expected scientific output. This flexibility could be utilized for VLBI experiments where a subset of antennas may commensally or independently participate with the AVN, EVN or Australian LBA. The same is true of two or more independent, MeerKAT-only experiments. Through a suite of simulations of the tied-array and interferometric performance of MeerKAT sub-arrays, this contribution presents initial results from a project that will investigate optimal sub-arraying strategies for a range of potential MeerKAT-VLBI and MeerKAT projects. We aim to systematically explore the scientific, technical, and processing trade-offs of MeerKAT sub-array and commensal observations, which is ultimately aimed at maximising the scientific utility of both MeerKAT and the VLBI networks it will participate in.

14th European VLBI Network Symposium \& Users Meeting (EVN 2018)

8-11 October 2018

Granada, Spain

\footnotetext{
*Speaker.
} 


\section{Introduction and Motivation}

The dense core of the MeerKAT array, which spans a distance of about $1 \mathrm{~km}$ in diameter, includes just over two thirds of the 64 antennas. The remaining antennas are located in the spiral arms that extend over a distance of $8 \mathrm{~km}$ in diameter. Each MeerKAT antenna is composed of a $13.5 \mathrm{~m}$ main reflector and a $3.8 \mathrm{~m}$ diameter sub-reflector in an Offset Gregorian optical layout. The MeerKAT array will be equipped with UHF (580 - $1015 \mathrm{MHz}$ ), L-band (900 - $1670 \mathrm{MHz})$, and S-band (1750 $3500 \mathrm{MHz}$ ) receivers; and potentially X-band $(8-14.5 \mathrm{GHz})$, if funding for these is secured. Given its high sensitivity and geographic location, the inclusion of the MeerKAT array into global VLBI networks could provide significant improvements in their scientific output, despite band overlap with current networks not being complete. A phased-up MeerKAT would have an effective area of a $\sim 110 \mathrm{~m}$ single dish antenna, with an SEFD of $\lesssim 10 \mathrm{Jy}$ at $1.4 \mathrm{GHz}$. This would add significant sensitivity to existing VLBI networks, especially in the longest baselines of $>7000 \mathrm{~km}$. Figure 1 illustrates the improvement in sensitivity MeerKAT-VLBI would add to the EVN.

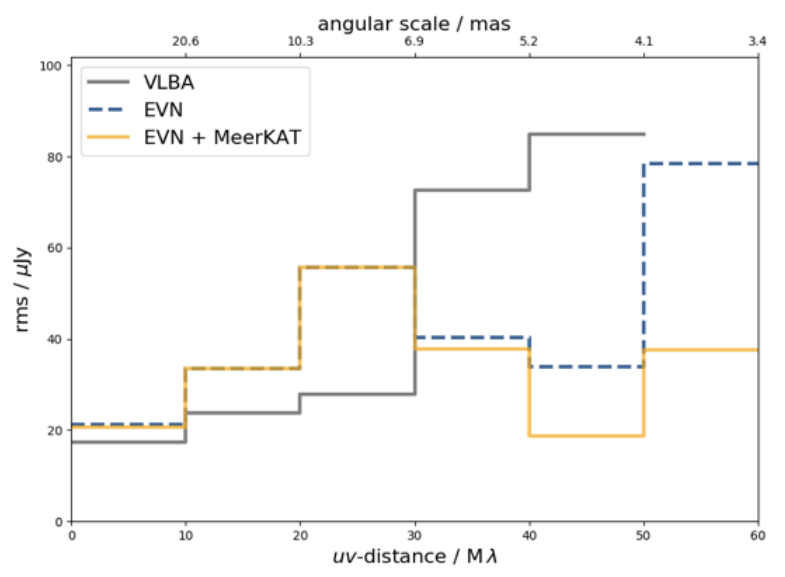

Figure 1: Demonstration of the enhancement of the EVN by MeerKAT (reproduced from Deane 2017). This shows a comparison of the VLBA, EVN and EVN+MeerKAT $18 \mathrm{~cm}$ performance towards the XMM-LSS extragalactic field (Dec. $=-4.8 \mathrm{deg}$ ). The plot shows the sensitivity per $u v$-bin in $10 \mathrm{M} \lambda$ increments, demonstrating the major improvement in $\gtrsim 40 \mathrm{M} \lambda$ spatial frequency range with the addition of MeerKAT, despite the lack of full band overlap (which is accounted for). MeerKAT sub-arrays could of course make scaled improvements of the above. A large increase in sensitivity could also be made with MeerKAT participation in the Australian LBA.

The over-arching objective of this project is to maximise the scientific utility of both MeerKAT and its contribution to existing VLBI networks. It aims to achieve this by exploring enhancement of VLBI performance by adding sub-arrays of MeerKAT antennas, and the resultant performance implication of standalone MeerKAT experiments. The compact core configuration accentuates the typical trade-off between image sensitivity and angular resolution which is controlled by the userspecified imaging weights (i.e., the robust parameter in Briggs weighting, Briggs, 1995). In Figure 2, we show this trade-off for an 8-hour MeerKAT observation, including the contribution of confusion noise. Instead of suppressing the statistical power of the vastly more numerous short 
baselines ( $\lesssim 1 \mathrm{~km}$ ) through low robust parameters $(\lesssim-0.5)$, one can conceivably achieve comparable performance through the removal a few antennas in the core and adjusting the MeerKAT experiment's imaging weights appropriately. Similarly, one could conceive of a pulsar search experiment in Open Time that discards a subset of the outermost antennas to reduce the real-time beamforming processing load. If either of these cases has a negligible impact on the scientific objectives (or a favourable impact on processing considerations) of the relevant MeerKAT observation, the removed antennas could be used in an independent MeerKAT experiment or alternatively be used to participate in a VLBI experiment with, for example, the AVN, EVN or LBA. We emphasize here that this is not approved SARAO policy/priority, but rather an exploratory exercise at this point in time.

\section{Quantifying the impact of sub-arraying for MeerKAT and MeerKAT-VLBI experiments}

While SARAO will decide the policy, capabilities, and priority of potential MeerKAT-VLBI operations in the future, we can envision three potential "primary MeerKAT-VLBI observing modes": (1) dedicated MeerKAT-VLBI experiments within the Open Time proposal allocation; (2) commensal VLBI with approved Large Survey Projects (LSPs, e.g. MIGHTEE, LADUMA) and other Open Time projects with pre-coordination between the relevant principal investigators; and (3) sub-arraying in Open Time with the same required pre-coordination. The third of these potential observing modes is the main focus of this study. In order to illustrate the potential for sub-arraying (and commensal) MeerKAT-VLBI, it is necessary to develop appropriate tools to visualize and quantify the effect of removing specific antennas from the MeerKAT array. Figure 2 shows level of the thermal noise, the confusion noise, and effective noise with different robust weight parameters.

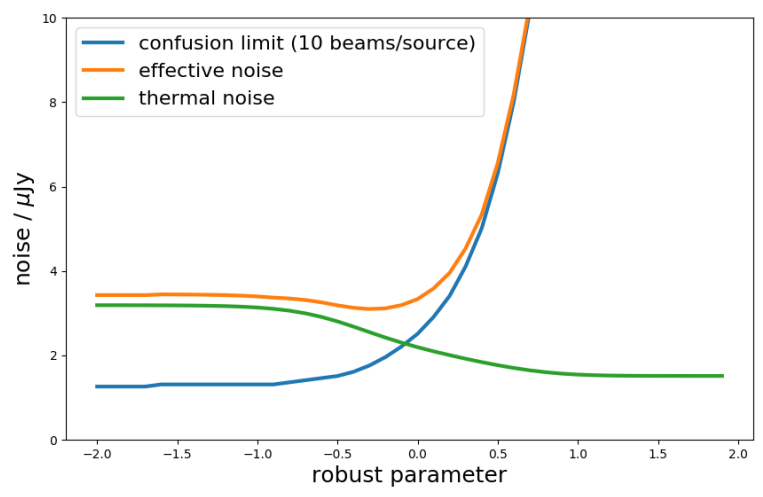

Figure 2: A plot of how the thermal noise $\left(\sigma_{\mathrm{t}}\right)$, the confusion noise, $\left(\sigma_{\mathrm{c}}\right)$ (assuming 10 beams/source and source counts from Bonaldi et al., 2019) and the effective noise $\left(\sigma_{\mathrm{e}}\right)$ vary as function of the robust image weighting parameter. The effective noise, $\sigma_{\mathrm{e}}=\sqrt{\sigma_{\mathrm{t}}^{2}+\sigma_{\mathrm{c}}^{2}}$, is mostly uniform for robust parameters $<0$ and it increases sharply for values $>0$. 


\section{Sub-Arraying Strategies}

In order to systematically explore different sub-arraying strategies, we will seek to split MeerKAT into sub-arrays using several different approaches. The most simple is selecting antennas by their distances from the array core, which is what we illustrate at this point, the very start of this project. Figure 3 illustrates the antenna configuration in terms of baseline lengths (towards Dec $=-30 \mathrm{deg}, 8$ hour observation) and antenna distance from core, which could be used to guide sub-array selection.
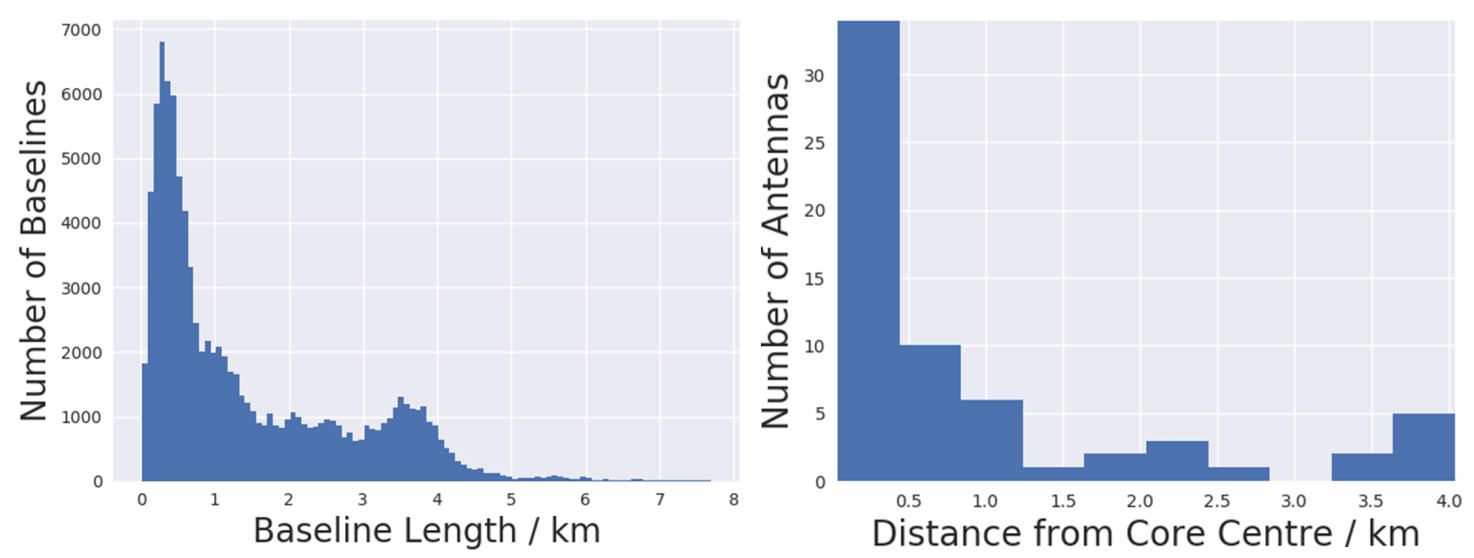

Figure 3: Left: The MeerKAT projected baseline length distribution for a 8-hour track of a source with $\mathrm{Dec}=-30 \mathrm{deg}$. The first peak represents the core-core baselines where most of the antennas are situated. The second smaller peak represents baseline lengths for "core-outlier" baselines Right: MeerKAT antenna distances from the array centre. The majority of the antennas are within the core which spans about $1.2 \mathrm{~km}$ from the array centre.

One of the ways that we can visualize the effect of removing certain antennas from the MeerKAT array is to plot the effective noise and PSF sizes of the sub-arrays that result from subtracting those antennas. Figure 4 shows heat maps of the effective noise and PSF size as a function of the robust imaging parameter for 64 hypothetical sub-arrays of MeerKAT. Each sub-array has all antennas included except for that one indicated on the y-axis tick label. The y-axis tick labels are sorted by their distance from the array core. Antennas towards the top correspond to sub-arrays with antennas removed from the core, while the bottoms correspond to the sub-arrays without an outer antenna. This assumes an 8-hour L-band observation of a source at Declination $=-30 \mathrm{deg}$. 

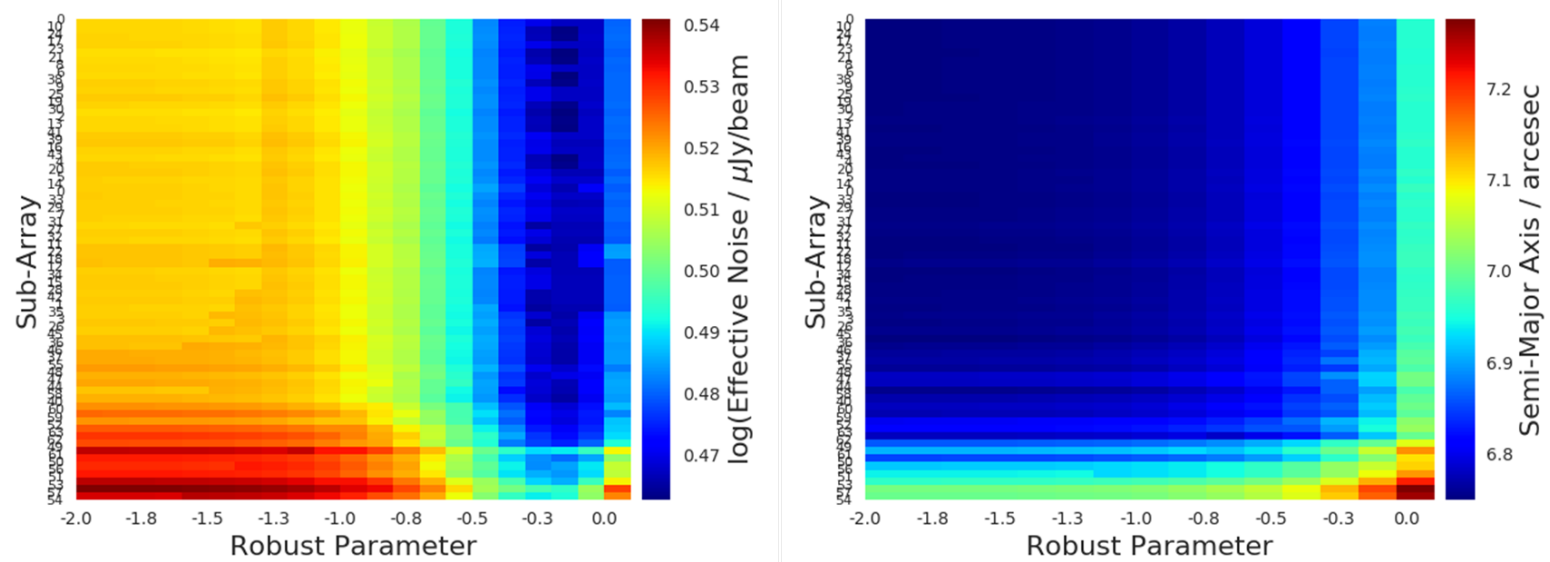

Figure 4: Effective noise and PSF size as a function of the robust parameter. Left panel: shows the Effective noise (color-scale) as a function of the robust parameter. We exclude robust parameters above 0.1 because the effective noise increases dramatically above this value, driven by the increase in confusion noise at the lower angular resolution that results from higher robust parameters (see Figure 2). Right panel: shows the PSF size (color-scale) for robust parameters for the same region covered on the left plot. The PSF size remains at an average of approximately $\sim 7(6.8$ - 8.4) arcsec for all sub-arrays for robust weight parameters less than 0.1. This serves to illustrate that removing a single antenna from the 64 MeerKAT array can potentially have a negligible effect for certain science cases, if the imaging weights are appropriately adjusted.

\section{Conclusion}

This new era of flexible backends and interferometers comprised of large numbers of antennas presents opportunities for intelligent sub-arraying strategies that can increase the scientific yield of these highly over-subscribed telescopes. In order to find optimal sub-arraying strategies, we require quantification of the relevant parameters, which is the primary focus of this project. In this contribution, we present an initial effort toward that goal for MeerKAT, with potential MeerKAT-VLBI opportunities as a possible beneficiary. A more complete analysis with these methods will be directly applicable to current and future $\mathrm{cm}$-wave and $\mathrm{mm}$-wave interferometers such as the SKA1-mid and ALMA. This is particularly true of arrays such as SKA1-mid where not all antennas would have the full suite of receiver bands installed.

\section{Acknowledgments}

I would like to acknowledge the funding from SARAO and the time they have allowed for me to further my studies. A great appreciation to the contribution of my supervisor Prof. Roger Deane for the guidance and extra effort to get the project to where it is. I also acknowledge the patience and love of my lovely wife Mrs Malebo Qwabe for supporting me in pursuing my MSc. even though it takes time away from our family. Thank you. 


\section{References}

[1] Briggs, D. (1995). High Fidelity Deconvolution of Moderately Resolved Sources. Ph.D. The New Mexico Institute of Mining and Technology, Socorro, New Mexico.

[2] Bonaldi et al.(2019). 2019MNRAS.482....2B Bonaldi, A., Bonato, M., Galluzzi, V., et al. 2019, mnras, 482,2

[3] Deane, R. (2016). Extragalctic VLBI surveys in the MeerKAT era. In: MeerKAT Science: On the Pathway to the SKA. Stellenbosch, South Africa: Proceedings of Science. 